\title{
Numerical hydrodynamics with a parallel integrated chemistry
}

\section{The interaction of a stellar jet with a molecular cloud}

\author{
A. J. Lim, J. M. C. Rawling, and D. A. Williams` \\ Department of Physics and Astronomy, University College London, Gower Street, London WC1E 6BT, UK
}

Received 6 March 2001 / Accepted 26 June 2001

\begin{abstract}
The interaction of a stellar jet with a molecular cloud is investigated using a computational approach in which the hydrodynamics, chemistry, and thermal behaviour are simultaneously and self-consistently integrated. The model incorporates the chemistry of 102 species whose abundances are coupled through a total of 1514 reactions. The dynamical and chemical structures are presented when the dynamics have achieved near steady-state. The results show that large variations in both density and temperature occur throughout the interaction region, and that these variations enable the identification of specific tracers of the various zones of interaction to be made. These tracer species are predicted to have detectable column densities and will have characteristic velocities and line profiles that should make them distinct from species in the ambient gas. Regions that should be chemically identifiable include the double shock structure at the jet head, the expansion of dense material from the jet head into the wake, the warm gas excited by the outer bow shock, and the mixing in the wake region.
\end{abstract}

Key words. ISM: clouds - ISM: jets and outflows - ISM: molecules

\section{Introduction}

Jets and collimated outflows are now accepted to be common phenomena in many astrophysical situations. In particular, jets from young stars or protostellar accretion structures and the closely associated Herbig-Haro (HH) objects are widely thought to be an integral part of the star formation process. In parallel with observational advances, the theoretical modelling of these regions has improved significantly over the past few years, primarily with the development of higher resolution numerical simulations and more complex chemical and radiative models. Consequently, some success has been achieved in predicting the dynamical and chemical behaviour of stellar jets.

In this paper we consider the dynamical and chemical behaviour of a HH-type jet head. This will typically be a jet which is more dense than its environment (although it may encounter regions of much higher density), and will have a high Mach number. Modelling of these jets has previously tended to concentrate on either the dynamics of the flow (see the review of Raga 2001 and references therein) or the general chemical aspects of shocks, although at least one attempt has been made at a fluid-dynamic simulation of a jet head with a small number (six) of chemical species and a very limited reaction

\footnotetext{
Send offprint requests to: D. A. Williams,

e-mail: daw@star.ucl.ac.uk

* Just to show the usage of the elements in the author field.
}

network; namely Raga et al. (1995) (hereafter RTCB95). The research in the cited paper was prompted by observations (and interpretation) of molecular emission of bowshock-type HH objects, but its chemical aspects were limited by the computational capabilities of the time. In the first paper of this series (Lim et al. 1999) we presented a 2D numerical hydrodynamic code, employing adaptive grid techniques, with the ability to integrate simultaneously an extensive chemical network with arbitrary numbers of species and reactions. This was applied to the problem of the chemical behaviour in a laminar boundary layer. In particular, we studied the quasi-steady state chemical and physical structures in a small portion of a shear interface between an atomic stellar wind and a cool diffuse cloud, in unprecedented combined dynamical and chemical detail. In this paper we apply the same model (with a much extended chemistry) to the situation of a jet head in a natural extension of RTCB95.

In addition to calculating the flow patterns at the head of the jet, we also follow a reasonably complex chemical network consisting of 100 species and 1514 reactions. The environmental gas is assumed to have a density comparable to the interclump gas of a clumpy molecular cloud. We also consider a jet moving into a denser (uniform) environment, as would be the case if a protostellar jet moves into a higher density region such a cloud. The interaction of a jet with a dense clump (of size comparable to the jet radius) will be considered in a later paper. 


\section{The model}

We have solved the familiar 2D Euler equations under assumptions of axi-symmetry. An important difference between our formalism of the chemical rate equations and that of RTCB95 is that we do not solve a separate continuity equation for the total mass density, rather, we solve a mass continuity equation for each chemical species. The momentum and energy equations are solved using the total mass density, i.e. the sum of all the chemical species. Our system of equations therefore reads as follows:

$$
\frac{\partial \rho_{\mathrm{j}}}{\partial t}+\frac{\partial}{\partial x}\left(\rho_{\mathrm{j}} v_{\mathrm{x}}\right)+\frac{\partial}{\partial r}\left(\rho_{\mathrm{j}} v_{\mathrm{r}}\right)=M_{\mathrm{j}}-\frac{\rho v_{\mathrm{r}}}{r}
$$

$$
\frac{\partial\left(\rho_{\mathrm{T}} v_{\mathrm{x}}\right)}{\partial t}+\frac{\partial}{\partial x}\left[\rho_{\mathrm{T}} v_{\mathrm{x}}^{2}+P\right]+\frac{\partial}{\partial r}\left[\rho_{\mathrm{T}} v_{\mathrm{x}} v_{\mathrm{r}}\right]=-\frac{\rho v_{\mathrm{x}} v_{\mathrm{r}}}{r}
$$

$$
\frac{\partial\left(\rho_{\mathrm{T}} v_{\mathrm{r}}\right)}{\partial t}+\frac{\partial}{\partial r}\left[\rho_{\mathrm{T}} v_{\mathrm{r}}^{2}+P\right]+\frac{\partial}{\partial x}\left[\rho_{\mathrm{T}} v_{\mathrm{r}} v_{\mathrm{x}}\right]=-\frac{\rho v_{\mathrm{r}}^{2}}{r}
$$

$$
\begin{aligned}
\frac{\partial}{\partial t}[E]+\frac{\partial}{\partial x}\left\{v_{\mathrm{x}}[E+P]\right\} & +\frac{\partial}{\partial r}\left\{v_{\mathrm{r}}[E+P]\right\} \\
& =-G-\frac{v_{\mathrm{r}}(E+P)}{r}
\end{aligned}
$$

where, $\rho_{\mathrm{j}}$ are the mass densities of the individual species, $\rho_{\mathrm{T}}$ is the sum of these densities, $v_{\mathrm{x}}$ and $v_{\mathrm{r}}$ are the velocity components in the axial (flow) and radial directions respectively, $E$ is the internal energy of the fluid (per unit volume) and $P$ is the pressure. $M_{\mathrm{j}}$ are source terms describing the chemical reactions and $G$ is an energy sink term due to radiative losses. The internal energy $E$ is given by

$$
E=\frac{1}{2} \rho_{\mathrm{T}}\left(v_{\mathrm{x}}^{2}+v_{\mathrm{r}}^{2}\right)+\frac{P}{\gamma-1} .
$$

where $\gamma$ is the ratio of the heat capacities. In these models we assume $\gamma$ is constant with a value of $5 / 3$. The energy sink term $G$ contains atomic cooling using a parameterised non-equilibrium function which considers the same processes as Biro et al. (1995), and $\mathrm{H}_{2}$ cooling using the method of Hollenbach \& McKee (1979).

For our computation of the time dependent chemistry and dynamics, we solve this system of equations to second order on a 2D binary adaptive grid using the Reefa linked grid algorithm (Lim \& Raga 1999). The grid has 5 levels of binary refinement between a minimum resolution of $32 \times 16$ points and maximum resolution of $513 \times 257$ points. The spatial extent of the grid is $5.13 \times 10^{15} \mathrm{~cm}$ in the axial direction and $2.57 \times 10^{15} \mathrm{~cm}$ in the radial direction. We implement the Flux-Vector Splitting scheme of Van Leer (1982) to integrate the equations.

Following RTCB95, we perform the calculation by placing the grid in a reference frame co-moving with the jet head, and allowing the system to relax to an approximately steady state. The boundary conditions of the computational domain are as follows: we impose a reflection condition on the cylindrical axis at $r=0$, an inflow condition with the environmental density and abundances on the right hand boundary and a free-flow condition at $2.57 \times 10^{15} \mathrm{~cm}$. The jet is injected on the left-hand side of the grid; for $r<r_{\mathrm{j}}$ an inflow condition is imposed with the jet parameters, for $r>r_{\mathrm{j}}$ material is allowed to flow freely off the left-hand side of the grid.

The chemical network consists of 102 atomic and molecular chemical species interconnected by 1514 reactions. These species are composed of the elements $\mathrm{H}, \mathrm{He}$, C, N, O, Na, Si \& S. The reactions and rate coefficients were primarily drawn from the UMIST "Rate95" database (Millar et al. 1997), although additional reactions appropriate to the high temperature post-shock regions were also included. (Additional reactions of particular significance include neutral-neutral reactions, collisional dissociations, dissociative recombinations, charge exchanges and negative ion reactions. Reaction data pertaining to the hydrogen chemistry, high excitation reactions and collisional dissociations were drawn from Rawlings, Drew \& Barlow 1993.) This ensured that there was sufficient reaction data to span the wide range of physical conditions that exist in the jet-cloud interface. Most collisional dissociation reactions are not efficient at low densities and consequently were suppressed. It was also assumed that $\mathrm{CO}$ and $\mathrm{H}_{2}$ are self-shielding against photodissociation at all positions and times.

As described above the mass density of each species is described by a density continuity equation which is an integral part of the dynamical calculation. This method allows the chemical abundances and reaction rates to be followed in each timestep of the calculation, providing a more realistic description of the chemistry than the more usual "post-processing" methods, in which the chemical network is applied to temperature/density structures obtained from a dynamical calculation and allowed to relax to a steady solution.

We choose the time step to be the minimum of: a) a fraction (typically 0.5 ) of the minimum cell-crossing time on the grid, b) the time taken to change the density of any species by two orders of magnitude, and c) the length of time in which $90 \%$ of the thermal energy of the gas would be lost to radiative cooling. We find that the chemical timestep (b) is almost always the smallest by far. We are currently implementing an operator splitting technique which will allow the chemistry, dynamics and thermal balance to be integrated separately, but consistently.

For all chemical species we impose a zero-point fractional abundance of $10^{-12}$. We assume for reasons of computational efficiency that a species a with fractional abundance below this value makes no significant contribution to the chemical network. All reactions involving the destruction of such a species are suspended until the abundance rises above the zero point once again.

For the total elemental abundance budgets, we use the "raw" cosmic abundances in the case of the (dust-free) jet gas. With the exception of the sodium abundance, these values are taken from Snow \& Witt (1996). For 
Table 1. Elemental abundances, relative to hydrogen; (i) in the jet, (ii) in the dark, low density environment, and (iii) in the illuminated $\left(A_{\mathrm{v}}=3\right)$ dense cloud.

\begin{tabular}{|l|lll|}
\hline & (i) & (ii) & (iii) \\
\hline $\mathrm{He}$ & 0.07 & 0.07 & 0.07 \\
$\mathrm{C}$ & $2.14 \times 10^{-4}$ & $2.14 \times 10^{-4}$ & $1.07 \times 10^{-4}$ \\
$\mathrm{~N}$ & $6.6 \times 10^{-5}$ & $6.6 \times 10^{-5}$ & $6.6 \times 10^{-5}$ \\
$\mathrm{O}$ & $4.57 \times 10^{-4}$ & $4.57 \times 10^{-4}$ & $4.57 \times 10^{-4}$ \\
$\mathrm{Na}$ & $1.8 \times 10^{-6}$ & $1.8 \times 10^{-7}$ & $1.8 \times 10^{-8}$ \\
$\mathrm{Si}$ & $1.86 \times 10^{-5}$ & $1.86 \times 10^{-8}$ & $1.86 \times 10^{-8}$ \\
$\mathrm{~S}$ & $1.23 \times 10^{-5}$ & $1.23 \times 10^{-6}$ & $1.23 \times 10^{-7}$ \\
\hline
\end{tabular}

the molecular environment that is being impacted by the jet we assume that significant depletion of the refractory elements into dust grains has occurred. In the denser environments that we have considered $\left(n>100 \mathrm{~cm}^{-3}\right)$, additional depletion of $\mathrm{C}, \mathrm{Na}$ and $\mathrm{S}$ is also assumed, consistent with the observed depletions in dense molecular clouds. The total abundances for these three phases are given in Table 1. The chemical initial conditions in the jet and the environment were established by assuming that static, equilibrium conditions prevail in each component. Note that this implicitly assumes that the chemical relaxation timescale is less than the time that is taken for the jet to travel from its source to the beginning of the grid - the jet is assumed to be chemically "mature". The numerically integrated equilibrium chemical abundances (for selected species) for both the jet and the environment phases are tabulated in Table 2. Abundances are given for each of the three models described below.

The cylindrically symmetric injected jet has a radius of $r_{\mathrm{j}}=5 \times 10^{14} \mathrm{~cm}$ and a temperature of $T_{\mathrm{j}}=100 \mathrm{~K}$. This jet moves into an ambient medium of temperature $T_{\mathrm{e}}=20 \mathrm{~K}$. We have computed three models, each with a jet speed of $50 \mathrm{~km} \mathrm{~s}^{-1}$, but varying the jet-to-environment number density ratio. The values we have chosen are: Model A, $n_{\mathrm{j}}=100 \mathrm{~cm}^{-3}, n_{\mathrm{e}}=100 \mathrm{~cm}^{-3}, A_{\mathrm{v}}=3$; Model B, $n_{\mathrm{j}}=50 \mathrm{~cm}^{-3}, n_{\mathrm{e}}=500 \mathrm{~cm}^{-3}, A_{\mathrm{v}}=3 ;$ Model C, $n_{\mathrm{j}}=50 \mathrm{~cm}^{-3}, n_{\mathrm{e}}=5 \mathrm{~cm}^{-3}, A_{\mathrm{v}}=100 . n_{\mathrm{j}}$ and $n_{\mathrm{e}}$ refer to the jet and environment density ratios, respectively. model A is our "standard" calculation, whilst model B represents a jet impacting a denser region of the cloud. Both models assume typical levels of extinction of the interstellar radiation field (ISRF). Model $\mathrm{C}$, which has lower densities and excludes the ISRF, is included for reference purposes - having similar parameter values to those adopted in RTCB95. Models A-C were integrated for 100, 205 and 105 years, respectively, before approximately steady configurations were reached. However, it should be noted that the detailed structures within the flow are time-dependent on timescales that are comparable to the age of the jet.

The value of the jet radius that we have chosen is somewhat small compared to that used in recent similar calculations (although it is within a factor of 5 of the value used by Stone \& Norman (1993) with reference to the HH34 jet). The reason for this choice is so that the cooling distance can just be resolved in a very high resolution calculation (performed as a test of the convergence of the solution). The bowshock cooling distance can be calculated with the formula of Cabrit (1995), and for model B this yields a value of $d_{\text {cool }} \approx 5 \times 10^{11} \mathrm{~cm}$. This distance is clearly not resolved on our grid. A calculation similar to model B with twice the above resolution gives virtually identical results, however the resolution of the cooling distance in this calculation was little better than in those we present. We therefore increased the resolution until $d_{\text {cool }}$ could just be resolved by a few points (computational limitations required that the chemistry be removed in this calculation). This produced results consistent with those of the recent resolution study of Krause \& Camenzind (2001), where a very similar simulation was studied at a variety of resolutions. Consequently, although the highly complex behaviour near the contact discontinuity is not resolved in our models, we can be confident that the general features of the flow - and in particular the flows into and out of the broad regions described in the next section - have converged at the resolution we have chosen.

One could resolve the cooling distance simply by reducing the density (as is done in RTCB95). However, our main motivation in this work is to predict astronomically observable molecular tracers of broad regions of the flow. Models of chemistry that are run at densities significantly lower than is realistic would not identify relevant tracers of realistic situations. Therefore, we have where necessary sacrificed dynamical computational considerations in favour of chemical ones. The basic fluid-dynamical situation that we consider has been very well studied, with many higher resolution studies available in the literature (see references quoted above). Consequently, for our study of the chemistry, we chose to select very general regions in the flow, with conditions determined largely by shock strength and initial densities, and which should be easily identifiable, at least in simulations. Although the structure of these regions will vary greatly depending on all the physical and numerical parameters of a given simulation, the jump conditions and fluid crossing times of these regions should remain roughly comparable - and it is these two properties which have the strongest dynamical effect on the chemical network. The temperature structure behind the shocks in our models is indeed unlikely to be that obtained in a converged solution, however our broad regions are (obviously) large compared to the cooling region which is limited to a few cells behind a shockfront. Although the temperature structure is not truly converged in this cooling region, its spatial extent is generally very small compared to our broad regions. Thus, one can assume that it is the conditions in these broad regions which will have the most significant effect on the local abundances.

In the next section we discuss the results of our simulations. 
Table 2. Initial fractional abundances (relative to $n_{\text {tot. }}=n_{\mathrm{H}}+2 n_{\mathrm{H}_{2}}$ ) in the jet (jet) and the undisturbed environment (env.) for selected species in the three models (A, B \& C) discussed in the text. The notation $a(b)$ signifies $a \times 10^{b}$.

\begin{tabular}{c|cccccc}
\hline species & jet(A) & env.(A) & jet(B) & env.(B) & jet(C) & env.(C) \\
\hline $\mathrm{H}$ & $2.01(-3)$ & $1.70(-3)$ & $4.62(-3)$ & $3.80(-4)$ & $4.79(-3)$ & $6.31(-3)$ \\
$\mathrm{H}^{+}$ & $3.24(-8)$ & $3.64(-7)$ & $1.55(-7)$ & $3.09(-8)$ & $9.55(-8)$ & $6.31(-6)$ \\
$\mathrm{C}$ & $7.65(-5)$ & $1.02(-5)$ & $1.02(-4)$ & $8.51(-7)$ & $6.31(-5)$ & $1.29(-4)$ \\
$\mathrm{C}^{+}$ & $1.53(-5)$ & $5.15(-6)$ & $7.08(-5)$ & $2.82(-7)$ & $5.62(-6)$ & $8.51(-5)$ \\
$\mathrm{CH}$ & $2.12(-8)$ & $9.75(-9)$ & $2.69(-8)$ & $1.32(-9)$ & $2.82(-8)$ & $1.20(-7)$ \\
$\mathrm{CH}^{+}$ & $2.68(-12)$ & $9.70(-13)$ & $7.94(-12)$ & $3.89(-14)$ & $1.02(-11)$ & $3.55(-11)$ \\
$\mathrm{C}_{2}$ & $4.50(-9)$ & $1.82(-9)$ & $1.82(-8)$ & $2.40(-11)$ & $2.57(-9)$ & $3.98(-8)$ \\
$\mathrm{CO}$ & $1.22(-4)$ & $9.15(-5)$ & $4.07(-5)$ & $1.05(-4)$ & $1.45(-4)$ & $1.58(-6)$ \\
$\mathrm{CN}$ & $2.36(-9)$ & $2.07(-9)$ & $3.24(-9)$ & $6.17(-10)$ & $1.38(-8)$ & $4.37(-9)$ \\
$\mathrm{N}$ & $6.30(-5)$ & $6.00(-5)$ & $6.61(-5)$ & $3.02(-5)$ & $6.03(-5)$ & $6.61(-5)$ \\
$\mathrm{NH}$ & $2.15(-10)$ & $4.87(-10)$ & $3.16(-10)$ & $2.88(-10)$ & $1.38(-9)$ & $2.75(-9)$ \\
$\mathrm{NH}{ }_{3}$ & $6.40(-12)$ & $7.65(-11)$ & $2.34(-12)$ & $1.55(-9)$ & $1.58(-10)$ & $1.17(-11)$ \\
$\mathrm{NO}$ & $6.60(-9)$ & $6.65(-8)$ & $2.82(-9)$ & $1.35(-7)$ & $1.05(-7)$ & $2.00(-8)$ \\
$\mathrm{O}$ & $3.34(-4)$ & $3.65(-4)$ & $4.17(-4)$ & $3.47(-4)$ & $3.09(-4)$ & $4.57(-4)$ \\
$\mathrm{O}_{2}$ & $2.56(-8)$ & $1.33(-7)$ & $1.05(-8)$ & $6.17(-7)$ & $5.75(-7)$ & $9.33(-9)$ \\
$\mathrm{OH}$ & $4.63(-8)$ & $2.14(-7)$ & $6.17(-8)$ & $1.66(-7)$ & $2.88(-7)$ & $2.00(-7)$ \\
$\mathrm{S}^{+}$ & $1.20(-5)$ & $1.19(-7)$ & $1.23(-5)$ & $9.77(-8)$ & $9.77(-6)$ & $1.20(-6)$ \\
$\mathrm{H}_{2} \mathrm{O}$ & $3.28(-8)$ & $1.17(-7)$ & $2.51(-8)$ & $2.75(-7)$ & $5.25(-7)$ & $6.61(-8)$ \\
$\mathrm{Na}{ }^{+}$ & $1.79(-6)$ & $1.78(-8)$ & $1.78(-6)$ & $1.78(-8)$ & $1.78(-6)$ & $1.74(-7)$ \\
$\mathrm{HCO}{ }^{+}$ & $2.08(-10)$ & $6.15(-10)$ & $2.00(-10)$ & $2.88(-9)$ & $1.48(-9)$ & $5.37(-11)$ \\
$\mathrm{HCN}$ & $2.42(-10)$ & $1.04(-10)$ & $1.78(-10)$ & $5.25(-11)$ & $3.31(-9)$ & $7.59(-10)$ \\
$\mathrm{HNC}$ & $1.05(-10)$ & $8.90(-11)$ & $1.23(-10)$ & $3.24(-11)$ & $1.58(-8)$ & $3.16(-9)$ \\
$\mathrm{HNO}$ & $1.92(-11)$ & $5.55(-11)$ & $5.62(-12)$ & $3.24(-10)$ & $2.04(-9)$ & $5.25(-10)$ \\
$\mathrm{CS}$ & $1.61(-8)$ & $7.55(-11)$ & $7.08(-9)$ & $4.37(-11)$ & $1.35(-6)$ & $2.63(-9)$ \\
$\mathrm{SO}$ & $1.71(-10)$ & $4.71(-12)$ & $2.24(-11)$ & $6.92(-11)$ & $1.82(-9)$ & $1.32(-11)$ \\
$\mathrm{SO}{ }^{+}$ & $2.09(-11)$ & $3.58(-12)$ & $1.29(-11)$ & $3.16(-11)$ & $1.51(-10)$ & $1.32(-12)$ \\
$\mathrm{OCS}$ & $1.14(-13)$ & $2.70(-15)$ & $1.15(-14)$ & $1.02(-13)$ & $2.82(-12)$ & $8.51(-16)$ \\
$\mathrm{SI}+$ & $1.85(-5)$ & $1.82(-8)$ & $1.86(-5)$ & $1.45(-8)$ & $1.74(-5)$ & $1.82(-8)$ \\
$\mathrm{SIH}$ & $1.56(-11)$ & $1.53(-14)$ & $3.98(-12)$ & $4.90(-14)$ & $6.61(-10)$ & $1.91(-13)$ \\
& $3.54(-8)$ & $3.59(-10)$ & $8.51(-9)$ & $3.72(-9)$ & $7.24(-7)$ & $1.07(-11)$ \\
\hline
\end{tabular}

\section{Results}

\subsection{Dynamical aspects}

In this section we describe our dynamical results and make a comparison with those of RTCB95. The cited paper contains an exhaustive analysis of the dynamical behaviour of the jet head and, therefore, we will not concentrate heavily upon this aspect of the results. However, in order to check the validity of our results, it is useful to compare the dynamical behaviour of our models with that of RTCB95 before moving on to describe the results of our parallel integrated chemistry.

In broad dynamical terms we find very similar behaviour to that of RTCB95, with material compressed between the jet and bow shocks and expanding adiabatically away from the jet axis $(r=0)$ to form the bow shock which accelerates environmental material that is typically several jet radii away from the stem. Figure 1 displays plots of results obtained from model A showing, $\log _{10}$ (total number density) (panel a), $\log _{10}$ (temperature) (panel b), $v_{\mathrm{x}}$ (panel c) and $v_{\mathrm{r}}$ (panel $\mathrm{d}$ ). In model $\mathrm{A}$ the jet and environment densities are equal and thus so are the strengths of the jet- and bow-shocks. The maximum temperature is $\approx 30000 \mathrm{~K}$ consistent with shocks of $25 \mathrm{~km} \mathrm{~s}^{-1}$. However, the maximum density is much higher than that found by RTCB95 due to catastrophic post-shock cooling (a result of the higher densities). This leads to a somewhat unstable stagnation region the structure of which (as noted by RTCB95) is highly time- dependent. The velocity plots (panels c and d) show this behaviour; despite the bulk of the flow in the stagnation region being outwards into the wake, panel (d) shows a significant flow towards the jet axis (due to compression of the jet stem by the high pressures in the wake). This results from a "kink" developing in the bowshock which will at future times lead to the 


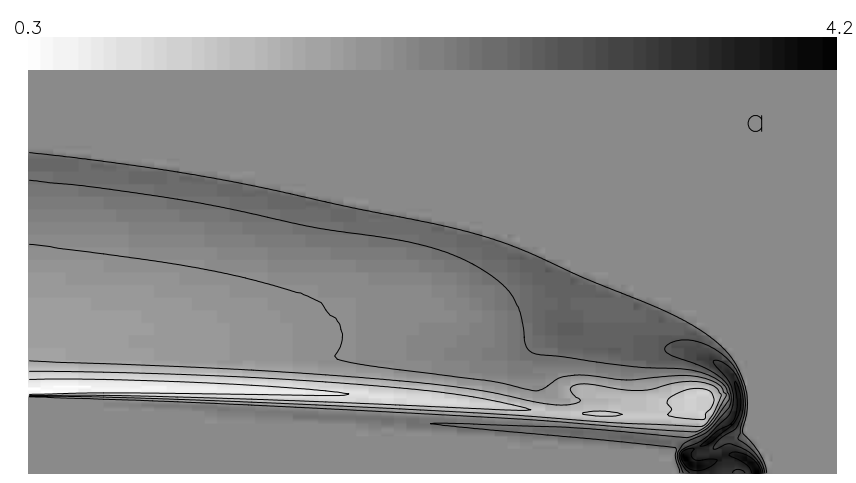

1.3
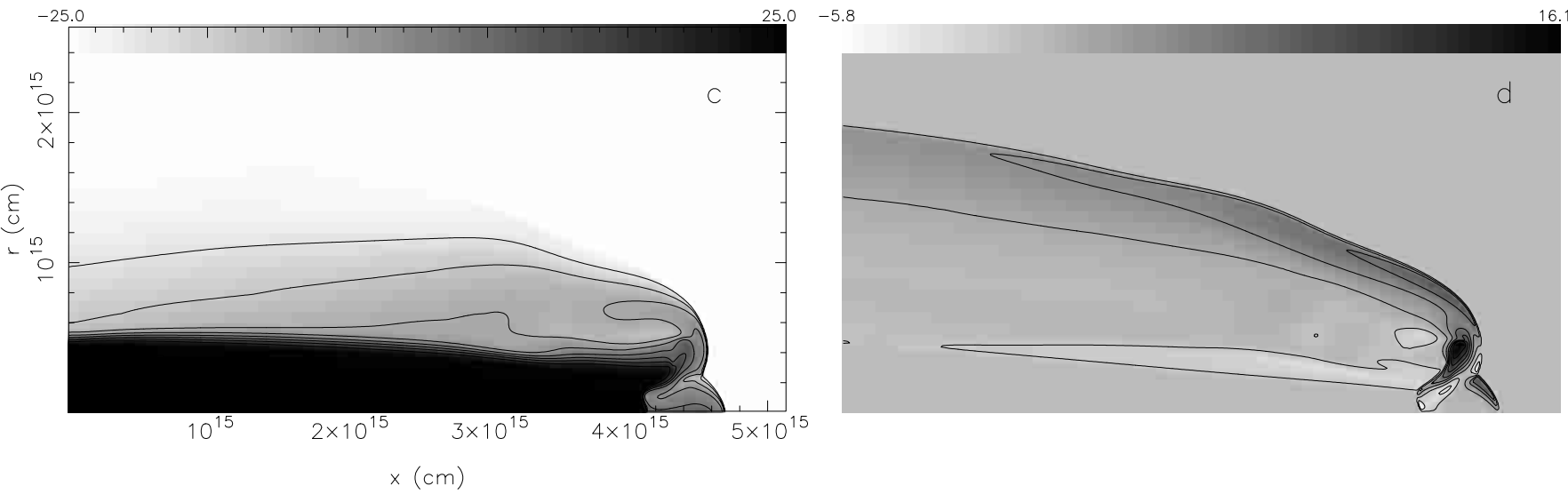

Fig. 1. The dynamical structure of the results from model A at time 110 years. The panels show the following data: a) log (total number density), b) $\log (T), \mathbf{c}) v_{\mathrm{x}}$ in $\mathrm{km} \mathrm{s}^{-1}$, and $\left.\mathbf{d}\right) v_{\mathrm{r}}$ in $\mathrm{kms}^{-1}$. Shown above the panels are the minima and maxima of the greyscales which run linearly between these extremes. There are ten contours equally spaced between the minima and maxima shown.

shedding of a vortex-like structure. It should be noted that these effects are not seen in model $\mathrm{C}$ (which has the closest initial conditions to those of RTCB95) as the densities are too low for such strong cooling of the postshock gas. The jet is surrounded by a high temperature, low density sheath of material which is in pressure equilibrium with the wake material. This region is composed of postjetshock gas (held against the jet-stem by the Bernoulli effect) and hot, mixed jet and environment material resulting from the shear boundary between the jet and the backwards-flowing wake. This second component was discussed in the first paper of this series (Lim et al. 1999) in which shear boundaries were studied in detail. This effect is only suggested in the temperature plot shown by RTCB95, and as also very weak in our "matching" calculation (model C) which has a similar temperature structure to RTCB95 along the edge of the jet (taking into account, of course, the difference in shock speeds).

A useful dimensionless parameter to compare is the post-bowshock "ridge-to-cavity" number density ratio, $\mu_{\mathrm{rc}}$. In our simulations we find the following values of $\mu_{\mathrm{rc}}$, model $\mathrm{A}: \approx 7.5$, model $\mathrm{B}: \approx 6.5$, model $\mathrm{C}: \approx 8$. These values are a factor of 2-3 lower than that found by RTCB95. Although our resolution is similar to that of RTCB95, there are several important differences which may be responsible for this variation of $\mu_{\mathrm{rc}}$. Primarily, we have used different parameters for our simulations with a much lower jet-speed and colder initial conditions, consequently the Mach numbers in our models are an order of magnitude greater than those of RTCB95. Secondly, the inclusion of a larger chemical network has a variable effect on the number density and thus the pressure-to-density ratio of the fluid.

The solution obtained by RTCB95 does however exhibit significant differences from our models, with more complex flow patterns and a collapse of the bowshock near the stagnation region. The reason for these differences is likely to be a combination of the diffusive effect of the small time step (which is determined by the chemistry), lack of resolution of the cooling distance and the effects described in the previous paragraph. Most importantly, the gas must cool over several iterations, which results in a more diffusive solution. A post-processing approach to the chemistry would alleviate these problems. However the principal uncertainty in the post-processing situation is how long the chemistry is exposed to the conditions in any given cell and post-processed models are therefore usually iterated to equilibrium. In our models, the chemical timescales are expected to be considerably longer than the dynamical timescales. In our Cases A and B, the chemical timescale is likely to be around 10000 years, while in Case $\mathrm{C}$ it may be longer than one million years. 


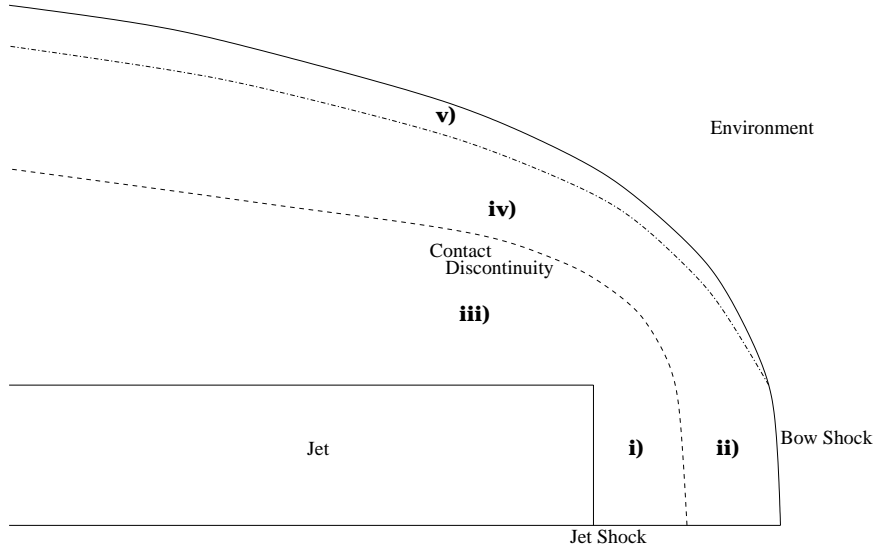

Fig. 2. A schematic diagram of the various zones of interaction described in the text.

Dynamical timescales in a $\mathrm{HH}$ jet head are typically of the order of decades and consequently we must use the parallel-integration approach.

We have varied the jet to environment density ratio in the three models presented. This is, in effect, equivalent to varying the relative strengths of the jet and bow shocks, since these parameters are related by a simple algebraic formula (see Cabrit 1995). In model A the strengths are equal, in model $\mathrm{B}$ the jet shock is stronger and in model $\mathrm{C}$ the bow shock is stronger. Model B shows very similar behaviour to model A with stronger cooling due to the higher environmental density. Model C shows much weaker cooling as the densities are very low compared with the other two models.

In the following section, we describe the effects of these variations on the chemical composition of the flow. The aim of this work is to identify tracers of the various zones of interaction. These zones, which are shown schematically in Fig. 2 are the:

(i) shocked jet gas;

(ii) shocked molecular cloud gas;

(iii) expanding shocked jet gas;

(iv) expanded shocked molecular cloud gas;

(v) post bow-shock environment gas.

Components (i) and (ii) appear at the head of the jet, while components (iii), (iv) and (v) constitute the wake of the jet/cloud interaction.

\subsection{Chemical aspects}

We discuss first model A, corresponding to unperturbed jet and environment densities of $n_{\mathrm{j}}=100 \mathrm{~cm}^{-3}, n_{\mathrm{e}}=$ $100 \mathrm{~cm}^{-3}$ respectively, a shock velocity of $50 \mathrm{~km} \mathrm{~s}^{-1}$ and in which the interstellar radiation field is obscured by 3 mag of dust extinction. The density and temperature structures illustrated in Figs. 1a,b show that between the two shocks (in the jet and in the environment) where the gas is compressed to high density, strong molecular cooling increases the density further and reduces the temperature. In models $\mathrm{A}$ and $\mathrm{B}$ the density in the stagnation region reaches more than $10^{4} \mathrm{~cm}^{-3}$ (due to the absence of strong cooling this is not seen in model $\mathrm{C}$ or in the calculation of RTCB95). The jet itself, however, is surrounded by the cylindrical shear layer between the jet stem and the wake, so that the jet remains a distinct feature in the interaction, at least until this layer can cool (see Lim et al. 1999). Outside this layer is a more extended wake of expanding shocked jet and environment gas in which the density and temperature are higher than in the undisturbed environment gas.

The model results show that the chemical network responds precisely to these widely varying physical conditions. Therefore, chemical abundances in the interaction zones also vary widely, and simple tracers of these zones can be readily identified.

Molecular species that trace the wake effectively, with abundances that are predicted to be detectable, include $\mathrm{CH}, \mathrm{CH}^{+}, \mathrm{H}_{2} \mathrm{O}, \mathrm{OH}, \mathrm{HCO}^{+}, \mathrm{NH}$, and $\mathrm{NH}_{3}$. In Fig. 3 we show the spatial distributions for several of these species: $\mathrm{CH}^{+}, \mathrm{H}_{2} \mathrm{O}, \mathrm{HCO}^{+}$, and $\mathrm{NH}_{3}$.

The plot for $\mathrm{CH}$ is similar to that for $\mathrm{CH}^{+}$(Fig. 3a). These species are enhanced, as to be expected, both in the warm sheath and in the weak shock at the outer boundary of the wake. They also trace quite precisely the two shocks at the head of the jet. Both species are present in the body of the wake, but at levels that may only marginally be detectable.

$\mathrm{H}_{2} \mathrm{O}$ (Fig. 3b) and $\mathrm{OH}$ (not shown) both delineate the double shock structure at the head of the jet. $\mathrm{OH}$ also traces the warm jet sheath and the outer boundary of the wake, but is of relatively low abundance at the jet sheath (where $\mathrm{H}_{2}$ itself is also of relatively low abundance). $\mathrm{H}_{2} \mathrm{O}$ occupies the wake and the space between the double shock structure at the jet-head with fairly high abundance, implying that much of the oxygen not locked in $\mathrm{CO}$ is converted to $\mathrm{H}_{2} \mathrm{O}$ in the body of the wake.

$\mathrm{HCO}^{+}$is well known as a tracer of interaction zones as well as of quiescent gas, and is seen here (Fig. 3c) to trace effectively the double shock structure and the outer boundary of the wake at significant abundance levels. It is also present at lower levels throughout the body of the wake.

$\mathrm{NH}_{3}$ (Fig. 3d) occupies both the wake and the double shock structure, and takes up a significant fraction of the available nitrogen. NH (not shown) has a similar distribution to that of $\mathrm{NH}_{3}$, but is somewhat more concentrated towards the outer boundary of the wake. It is remarkable that the $\mathrm{NH}$ and $\mathrm{NH}_{3}$ abundances are similar in the wake. NO is also distributed along the outer boundary of the wake, but at levels that are rather low for detection, although its abundance is high in the jet head.

Notably, all of the species mentioned above have abundances in the wake that are significantly enhanced over the values in both the jet and surrounding molecular environment, suggesting that they may be good tracers of jet/cloud interface activity.

The double shock region at the jet head is traced by many species created in post-shock gas, including those already mentioned. However, there are also other tracers 
$-9.7$

a
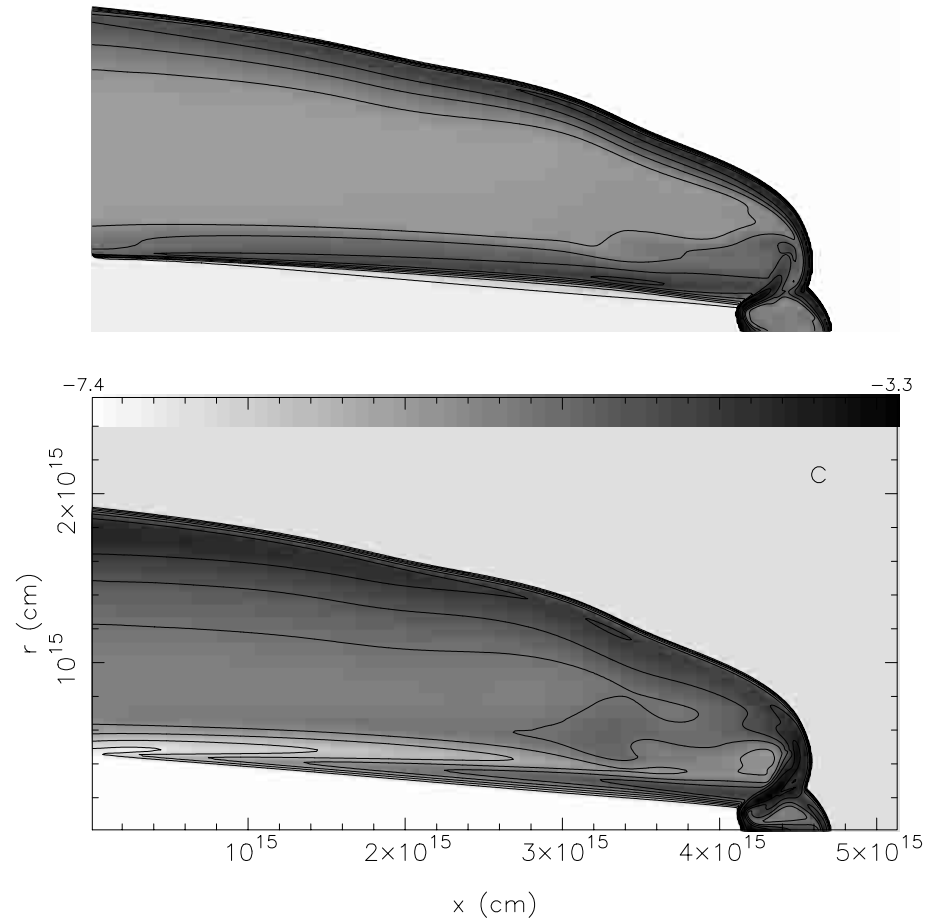

$-5.2$

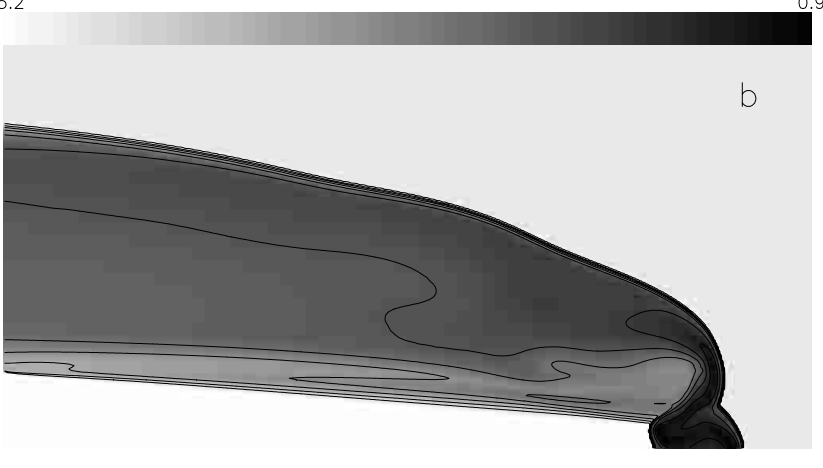

$-8.9$

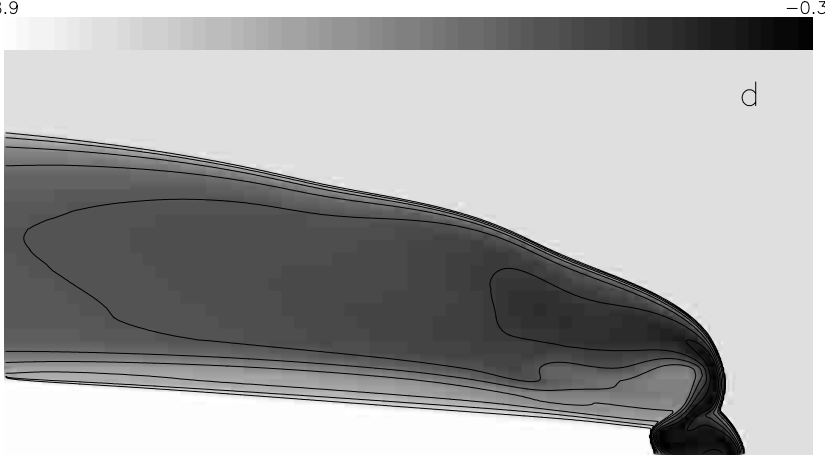

Fig. 3. As Fig. 1, but showing $\log _{10}$ (number density) of the following species: a) $\left.\mathrm{CH}^{+}, \mathbf{b}\right) \mathrm{H}_{2} \mathrm{O}$, c) $\mathrm{HCO}^{+}$, and d) $\mathrm{NH}_{3}$.

of the dense, colder gas between the two shocks (which results from the strong postshock cooling), and these are the species that are more characteristic of conditions in cold, quiescent, interstellar clouds; they include CO, CS, HCN, NO, and SO. Of these, CO (not shown) generally takes up most of the available carbon, and traces the density variations over the interaction region. $\mathrm{CO}$ is therefore most prominent in the double shock jet head region, and traces the beginning of the expansion of this compressed material into the wake. The velocity plot (Fig. 1d) shows that significant velocity shifts (on the order of $10 \mathrm{~km} \mathrm{~s}^{-1}$ ) may be associated with this gas, and so CO emission from this cold dense gas may be detectable as a distinct velocity feature against $\mathrm{CO}$ emission from the ambient gas.

The distributions of some of the remaining species, CS, HCN, NO and SO, are illustrated in Fig. 4. The patterns of these distributions look fairly similar, although the different species peak in different regions of the flow. The concentration of gas between the two shocks at the head of the jet is clearly evident in all these species, and they have abundances of $10^{-2}-10^{-3} \mathrm{~cm}^{-3}$ which should generate detectable column densities of more than $10^{11} \mathrm{~cm}^{-3}$ for the expected spatial dimensions of these interaction structures. In all cases, the initial flow of this compressed gas into the wake region should be evident - if not also spatially resolved, then at least from the velocity structure. However, in most cases, the abundances of these species in the wake are too low for easy detection - although significant abundances of $\mathrm{NO}$ are formed behind the weak bow shock (zone v).
There are several other species about which a few remarks are necessary. Both $\mathrm{C}$ and $\mathrm{C}^{+}$"avoid" the wake region. For the initial conditions adopted in these calculations, $\mathrm{C}$ and $\mathrm{C}^{+}$are abundant in the jet and environment gases but are converted to hydrocarbons in the wake. Obviously, $\mathrm{H}$ and $\mathrm{H}_{2}$ follow closely the density distribution shown in Fig. 1a; the $\mathrm{H}$ abundance is between $1-10 \%$ of that of $\mathrm{H}_{2}$. Throughout much of the wake not restricted to the wake boundary $-\mathrm{H}_{2}$ is present with a number density on the order of $10^{2} \mathrm{~cm}^{-3}$ at temperatures around $10^{3} \mathrm{~K}$. There should be copious emission from $\mathrm{H}_{2}$ in the $1-0$ vibrational band. $\mathrm{H}_{3}^{+}$is present in the jet, the environment, and the wake, at marginally detectable abundances. However, it traces the shocked environment at the jet head very well, in potentially detectable amounts. Its role in the chemistry is, however, rather more limited in this high excitation environment than in cool molecular clouds. Interestingly, atomic sulphur is abundant in the double shock jet head region, although its abundance is low in all other parts of the flow. This may simply reflect the high initial abundance of atomic sulphur in the jet. The relatively short timescale for the sulphur chemistry at low temperatures may help to explain its relative absence downstream from the shocks. Finally, the level of ionization in the gas, as measured by the $\mathrm{H}^{+}$abundance (see Fig. 5a), is high in the jet and environment and low in the wake where the rapid chemistry induced by the warm gas reduces the charge state through fast dissociative recombinations of molecular ions. In fact, the mixing of the environment gas into the wake, illustrated through 
$-7.8$

a
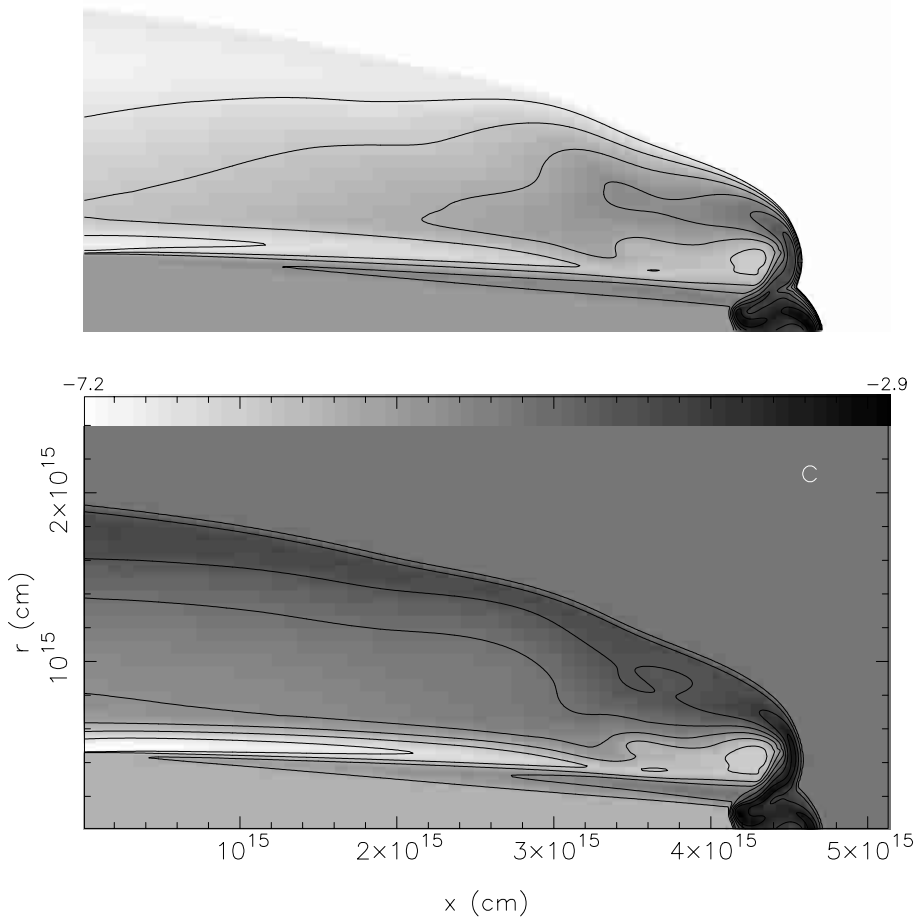

$-7.7$

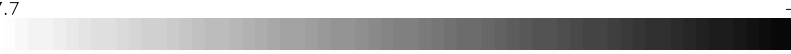

b
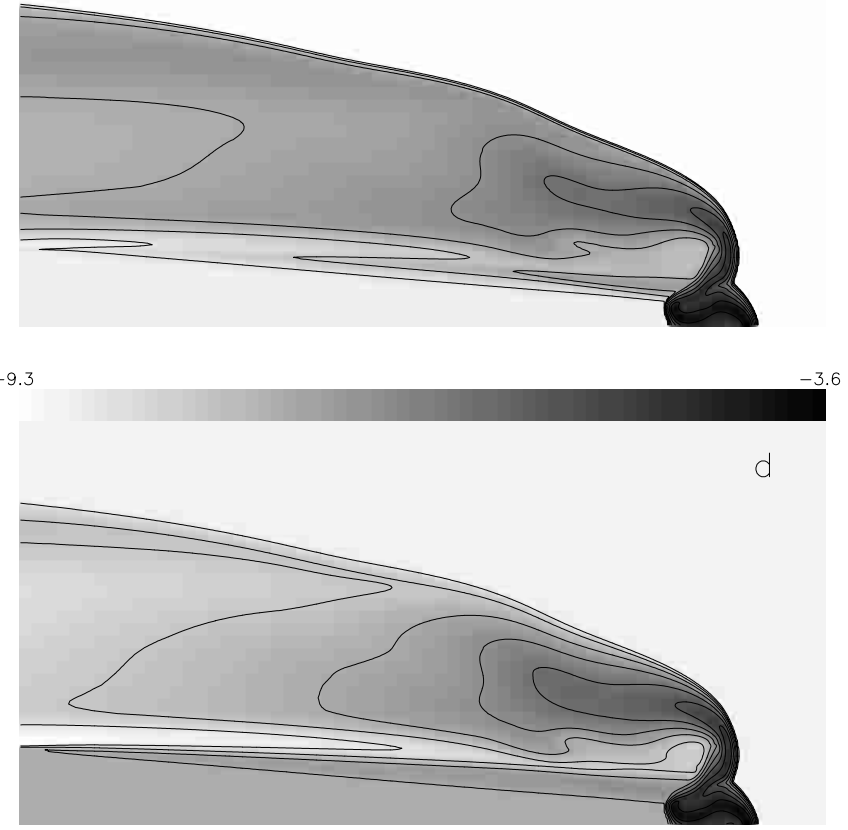

Fig. 4. As Fig. 1 but showing: a) CS, b) HCN, c) NO, and d) SO.

a notional "dye" (advected with the flow), is rather similar to the $\mathrm{H}^{+}$distribution (see Fig. 5b). Evidently, the characteristic chemistry of the wake, described here, is a consequence of the mixing of the initially compressed gas at the double shock region of the jet head, entrained into the wake, with the shocked environment gas.

In summary, detectable column densities $(N>$ $10^{11} \mathrm{~cm}^{-2}$ ) are predicted in the jet head region (zones i and ii) for most of the molecular species that we have considered in our chemistry, including $\mathrm{CH}, \mathrm{CH}^{+}, \mathrm{NH}, \mathrm{NH}_{3}$, $\mathrm{OH}, \mathrm{CO}, \mathrm{NO}, \mathrm{CS}, \mathrm{SO}, \mathrm{HCO}^{+}, \mathrm{H}_{2} \mathrm{O}, \mathrm{HCN}$ and vibrationally excited $\mathrm{H}_{2}$. Of these, all but CS, SO and HCN should be detectable in the wake (zones iii, iv and v). Identifiable emission from the post bow-shock environment (zone v) should be detectable for $\mathrm{CH}, \mathrm{CH}^{+}, \mathrm{NH}$, $\mathrm{OH}, \mathrm{CO}, \mathrm{NO}, \mathrm{HCO}^{+}, \mathrm{H}_{2} \mathrm{O}$ and $\mathrm{H}_{2}$. $\mathrm{CH}, \mathrm{OH}, \mathrm{HCO}^{+}$\& $\mathrm{H}_{2}$ are expected to be particularly prominent in this region (as compared to the bulk of the wake; zones iii and iv). In addition, the hydrides $\mathrm{CH}, \mathrm{NH} \& \mathrm{OH}$ (and also possibly $\mathrm{CH}^{+}, \mathrm{CO}, \mathrm{HCO}^{+} \& \mathrm{H}_{2} \mathrm{O}^{+}$) have observable abundances in the shear layer surrounding the jet stem.

\subsection{Comparisons with the other models}

We compare the model A results with two other models for which we have results: model B $\left(n_{\mathrm{j}}=50 \mathrm{~cm}^{-3}\right.$, $\left.n_{\mathrm{e}}=500 \mathrm{~cm}^{-3}, A_{\mathrm{v}}=3\right)$ and model $\mathrm{C}\left(n_{\mathrm{j}}=50 \mathrm{~cm}^{-3}\right.$, $n_{\mathrm{e}}=5 \mathrm{~cm}^{-3}$, radiation excluded). The jet velocity is the same, $50 \mathrm{kms}^{-1}$, in all three models. model $\mathrm{C}$ was explored as it is close to the situation investigated by
RTCB95, although our shock speeds are lower (due to the fact that our chemistry is not valid at the temperatures typically found at the head of the $130 \mathrm{kms}^{-1}$ jet studied in the cited paper) and the Mach numbers are significantly higher (due to our colder initial conditions). In fact, the dynamical results we have obtained are very similar to those of RTCB95. Here, we comment on the chemical differences between our three models.

Figures $6 \mathrm{a}-\mathrm{c}$ show the $\mathrm{H}_{2}$ distributions in the three models, from which it can be seen that there are significant differences in the dynamical behaviours of the three models at the jet head. However, the most pronounced change is in the wake which is wider and warmer when the environment density is low (model C) than when it is high - a consequence of inefficient post-shock cooling at lower densities. The jet sheath is also less pronounced in model $\mathrm{C}$ as compared to models $\mathrm{A}$ and $\mathrm{B}$. However, it should be noted that although the wake is more extended, the total column densities are generally lower in model $\mathrm{C}$ as compared to models $\mathrm{A}$ and $\mathrm{B}$ (which are dynamically and chemically quite similar).

The gas density significantly affects the chemistry, so that the tracer molecules are distributed differently in the three models. In Figs. 7a,b we illustrate this point by showing the $\mathrm{CH}^{+}$distributions for models $\mathrm{B}$ and $\mathrm{C}$, to be compared with Fig. 3a for model A. The effects of density and temperature combine to make the three distributions quite different in appearance. In model $\mathrm{C}, \mathrm{CH}^{+}$is abundant throughout the wake, while in model $\mathrm{B}$ it is largely associated with the warm sheath around the jet, although 

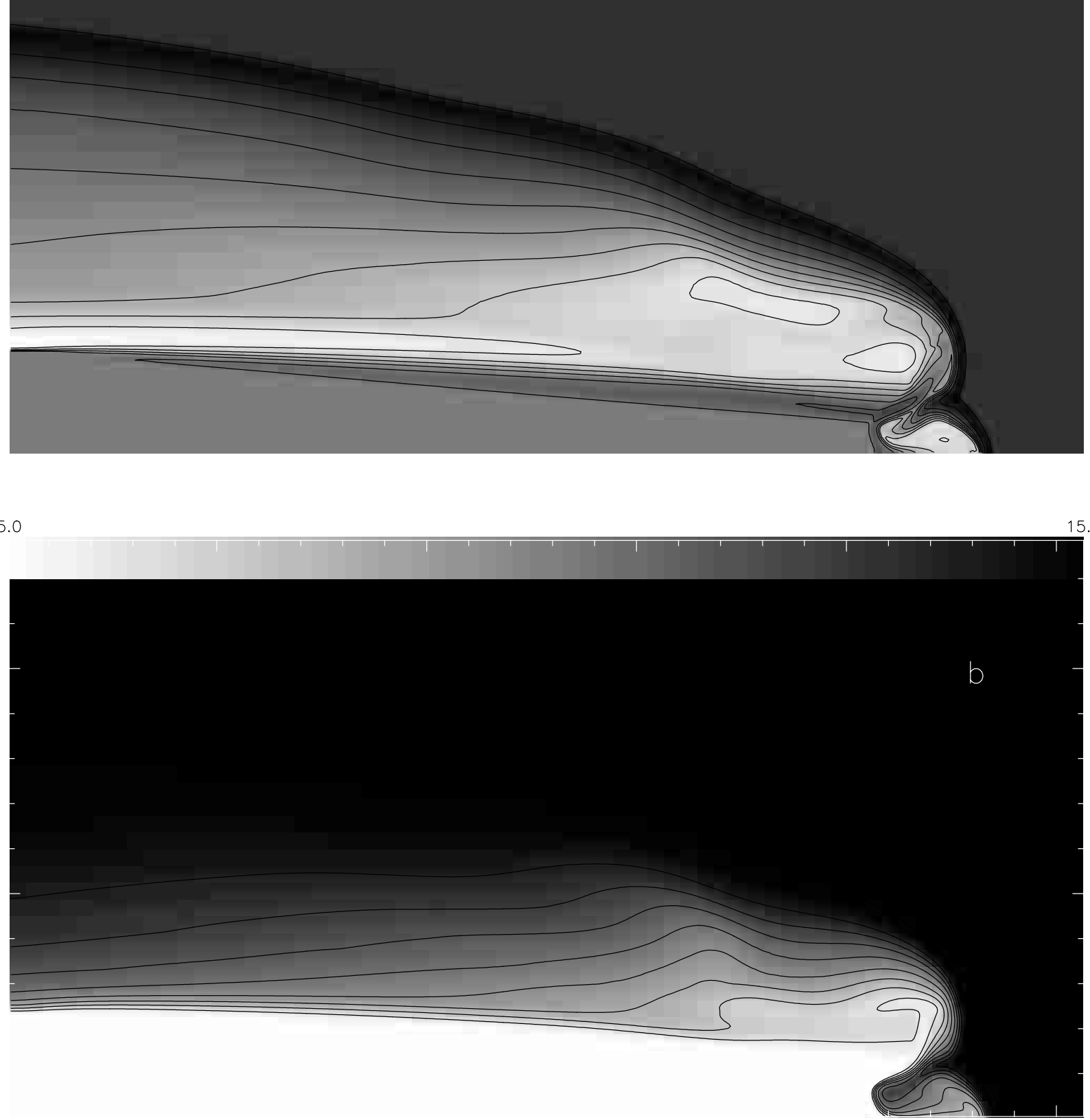

Fig. 5. As Fig. 1 but showing: a) $\mathrm{H}^{+}$, b) the numerical tracer ("dye") which has a value of 5 in the jet and 15 in the environment.

in model B it is not as prominent in the post bow-shock gas (zone $\mathrm{v}$ ) as it is in model $\mathrm{A}$.

In model $\mathrm{B}$ (Fig. 7c) $\mathrm{NH}_{3}$ occupies nearly the entire region in the double shock structure at the jet head, and fills nearly uniformly the relatively dense wake outside the warm jet sheath. This distribution is similar to that found in model A (see Fig. 3d). In model C (Fig. 7d), by contrast, the high density tracer $\left(\mathrm{NH}_{3}\right)$ is preferentially located near the strong shock at the jet head (though not so much at the outer shock) and in the densest part of the expanding wake which lies just outside the shear layer surrounding the jet.

Unlike model A, in model $\mathrm{B}, \mathrm{CH}, \mathrm{CH}^{+}, \mathrm{OH} \& \mathrm{HCO}^{+}$ all have undetectable post bow-shock abundances (i.e. in zone v). Indeed, the $\mathrm{OH}$ and $\mathrm{HCO}+$ abundance distributions in the wake mirror their behaviours in model A, so that in model $\mathrm{B}$ both species are strongly enhanced in the bulk of the wake (zones iii and iv). In model $\mathrm{B}, \mathrm{H}_{3}^{+}, \mathrm{NH}_{3}$, 


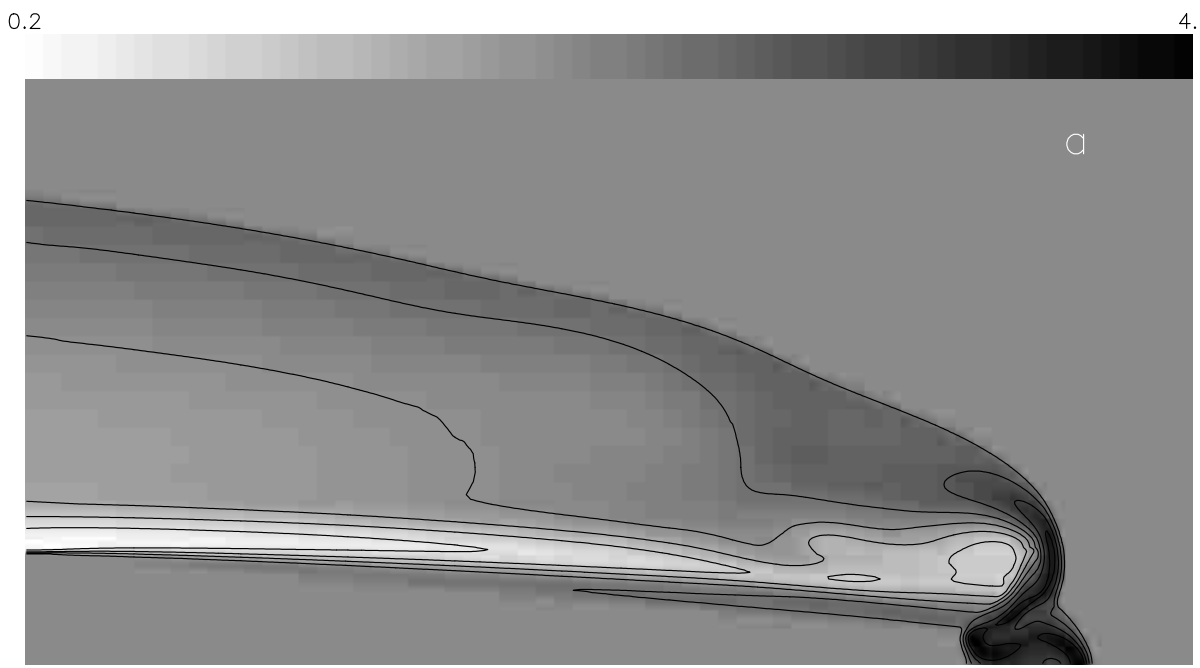

0.3

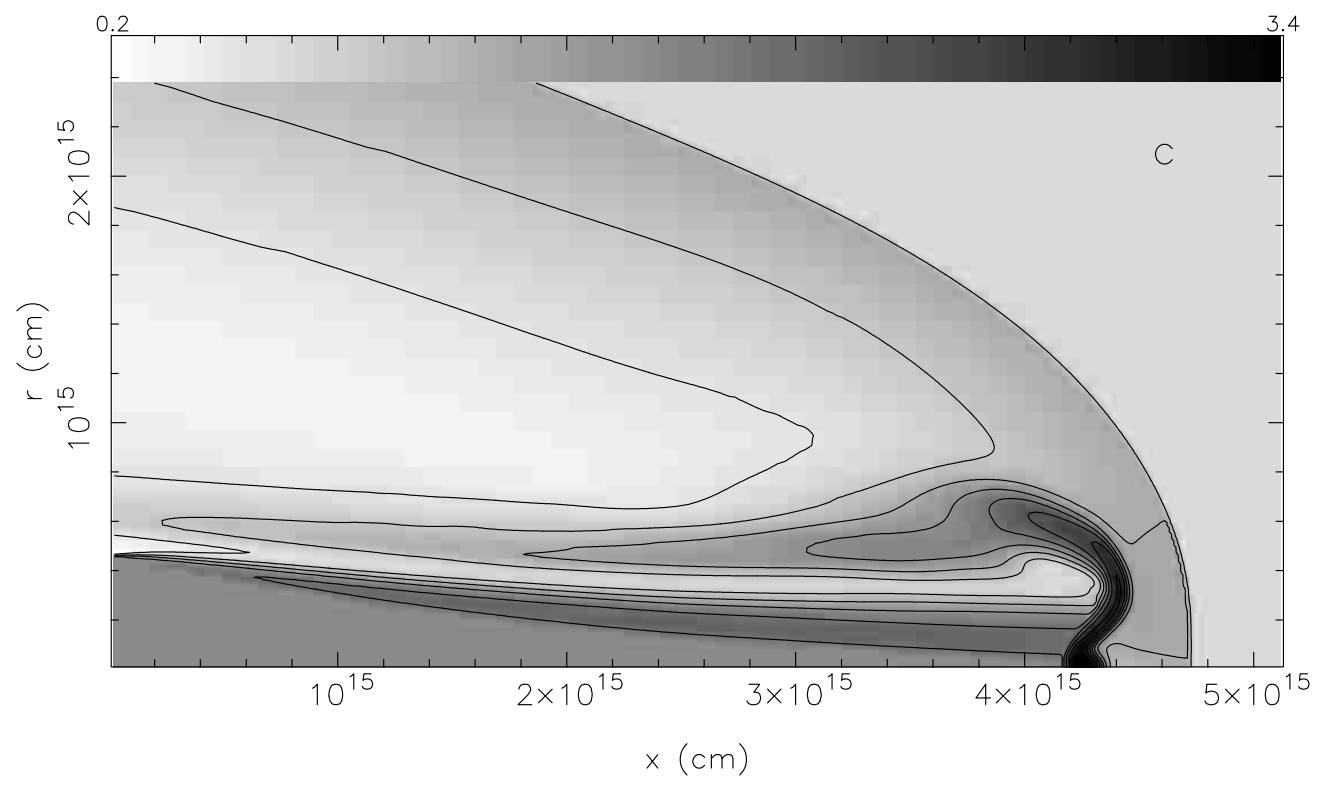

Fig. 6. Greyscales of $\log _{10}\left[n\left(\mathrm{H}_{2}\right)\right]$ for each of the models: A (panel a)), B (panel b)), and C (panel c)). The output times of models $\mathrm{B}$ and $\mathrm{C}$ shown here are 225 years and 105 years, respectively. 
$-10.7$
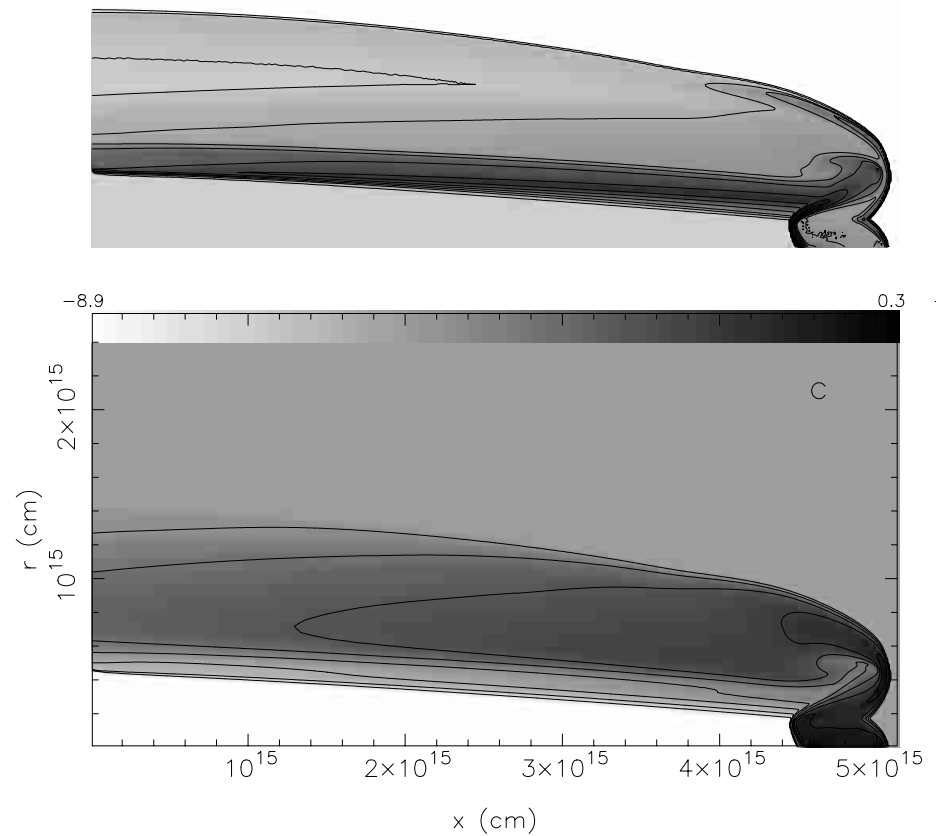

9.4

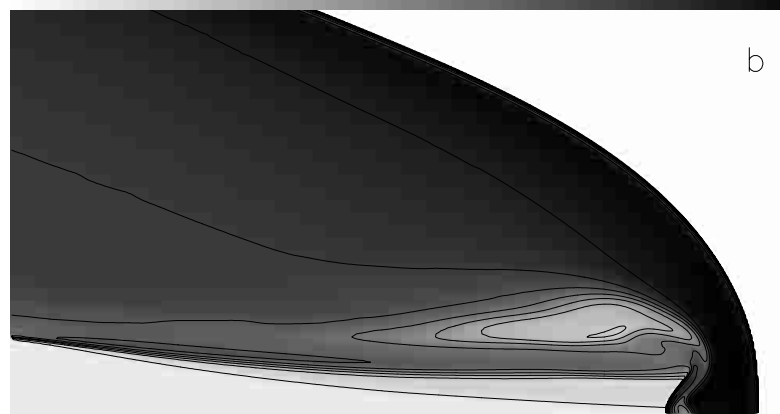

$-9.9$

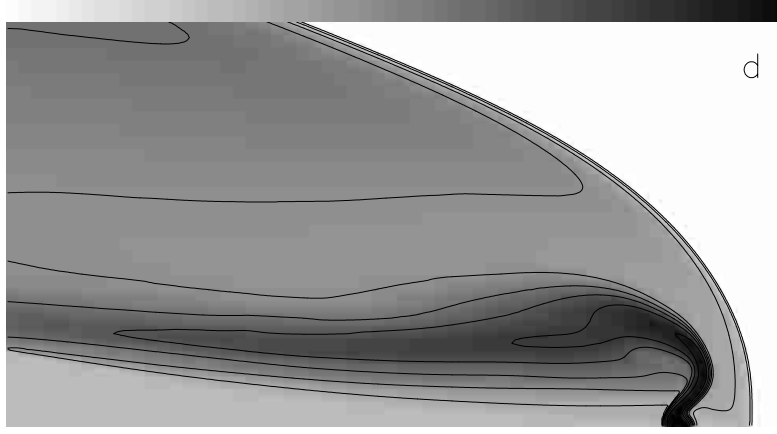

Fig. 7. Comparison for model $\mathrm{B}$ and model $\mathrm{C}$ of the abundances of $\mathrm{CH}^{+}$(panels a) and b), respectively) and $\mathrm{NH}_{3}$ (panels c) and $\mathbf{d}$ ), respectively).

$\mathrm{CS} \& \mathrm{H}_{3} \mathrm{O}^{+}$have abundances that are typically $2-10 \times$ smaller compared to model A, whilst the abundances of $\mathrm{SO}$ and $\mathrm{HCO}^{+}$are $\sim 5-8 \times$ larger.

As a consequence of the extended low density, high temperature post-shock region in model $\mathrm{C}$, the ions $\mathrm{H}^{+}$, $\mathrm{C}^{+}, \mathrm{H}_{3}^{+}, \mathrm{OH}^{+} \& \mathrm{H}_{2} \mathrm{O}^{+}$are more prominent, whilst many neutral molecular species $\left(\mathrm{H}_{2}, \mathrm{H}_{2} \mathrm{O}, \mathrm{HCN}, \mathrm{CS} \& \mathrm{SO}\right)$ are suppressed in the jet head (zones i and ii) as compared to model A. However, $\mathrm{CH}$ is enhanced to a detectable level in all of the wake components (zones iii, iv \& v) and $\mathrm{CH}^{+}$ is prominent in all of the jet-head and wake components in model $\mathrm{C}$. By contrast, $\mathrm{NH}_{3}, \mathrm{CO}$ and $\mathrm{NO}$ are relatively suppressed in the shocked cloud components (zones ii, iv and $\mathrm{v}$ ).

These examples serve to illustrate that molecular tracers may be found to identify not only the various regions of the flow, but also to distinguish between different physical conditions that may apply in different cases.

\section{Conclusions}

Using a self-consistent model that integrates simultaneously the equations of hydrodynamics, chemistry, and thermal balance, we have investigated computationally the dynamical and chemical structures established when a fast stellar jet impacts on a molecular cloud. A fairly complex set of chemical reactions was included in the description and followed during the dynamical computations.
The main dynamical features of our simulations agree well with previous studies of jets and are as follows:

1) Gas is compressed to high density between the bow shock and the jet shock. If the density is sufficiently high, subsequent molecular cooling leads to further strong compression; 2) this high density gas tends to expand into the wake, sometimes through vortex shedding, the wake is a mixing region of shocked environment gas and shocked jet gas; 3) the dense gas tends to cool rapidly, so that the temperature and density distributions are near-inverse images of each other; 4) the jet is encased in a shear boundary layer of warm gas; 5 ) the bow shock expands laterally into the environment gas, to several jet radii.

The chemical implications of this study are: 1) the density and temperature variations throughout the interaction zone create an environment in which a rich chemistry occurs, and with considerable chemical variation allowing the identification of particular tracers of specific zones; 2) the tracers should have abundances that constitute detectable column densities, and should have specific velocities and line profiles; 3 ) interaction zones that should be identifiable through these tracers include the doubleshock structure at the jet head, the expansion of dense gas into the wake, the mixed gas in the body of the wake, and the gas excited by the bow shock; 4) the width of the wake extends to several jet radii, and is sensitive to the ambient gas density, with lower densities implying a wider but more tenuous and less chemically-rich wake. 
This is mainly due to molecular cooling which, at the higher densities, rapidly lowers the postshock temperatures into a more chemically favourable regime.

An important process, not considered here, is the effect of radiation from the postshock regions. Work is currently in progress to include the effects of time- dependent radiative transfer in simulations using the Reefa grid. This will allow the effect of the radiation field resulting from the jet-cloud interaction to be self- consistently included in the chemical network.

In this study we have identified a number of molecular tracers of all of these interface regions. For the jet and environment parameters that we have considered these include $\mathrm{CH}, \mathrm{CH}^{+}, \mathrm{NH}, \mathrm{NH}_{3}, \mathrm{OH}, \mathrm{CO}, \mathrm{NO}, \mathrm{CS}, \mathrm{SO}, \mathrm{HCO}^{+}$, $\mathrm{H}_{2} \mathrm{O}, \mathrm{HCN}$ and vibrationally excited $\mathrm{H}_{2}$. Moreover we have shown that the relative distribution of these tracers is strongly coupled to the physical conditions within both the jet and the surrounding medium that the jet impacts. Evidently, observational studies of jet/cloud interactions should reveal much detail about the local physical conditions within both media.

Acknowledgements. A. J. Lim acknowledges support from a PPARC postdoctoral associateship and D. A. Williams is grateful for the award of a PPARC Senior Fellowship.

\section{References}

Biro, S., Raga, A. C., \& Cantó, J. 1995, MNRAS, 275, 557

Cabrit, S. 1995, Shocks in Astrophysics, ed. T. J. Millar, \& A. C. Raga (Kluwer: Dordrecht), 81

Hollenbach, D., \& McKee, C. F. 1979, ApJS, 41, 555

Krause, M., \& Camenzind, M. 2001, Emission Lines from Jet Flows - Poster Proceedings, Isla Mujeres, Mexico, ed. P. F. Velázque, \& R. González, 22

Lim, A. J., \& Raga, A. C. 1999, MNRAS, 303, 546

Lim, A. J., Rawlings, J. M. C., \& Williams, D. A. 1999, MNRAS, 308, 1126

Millar, T. J., Farquhar, P. R. A., \& Willacy, K. 1997, A\&AS, 121,139

Raga, A. C. 2001, Emission Lines from Jet Flows, Isla Mujeres, Mexico, ed. W. Henney, \& W. Steffen, 22

Raga, A. C., Taylor, S. D., Cabrit, S., \& Biro, S. 1995, A\&A, 296,833

Rawlings, J. M. C., Drew, J. E., \& Barlow, M. J. 1993, MNRAS, 265, 968

Snow, T. P., \& Witt, A. N. 1996, ApJ, 468, L65

Stone, J. M., \& Norman, M. L. 1993, ApJ, 413, 198

Van Leer, B. 1982, Lect. Notes. Phys., 170, 507 